\title{
Preguntas sobre alimentación y nutrición planteadas en los libros de texto de Ciencias de la Naturaleza en Educación Primaria
}

\author{
Cristina Gil González (D) , Ángel Luis Cortés Gracia iD \\ Departamento de Didácticas Específicas. Área de Didáctica de las Ciencias Experimentales. Universidad de \\ Zaragoza.Zaragoza.España.crisgilgon@unizar.es,acortes@unizar.es
}

[Recibido: 2 diciembre 2020. Revisado: 7 febrero 2021. Aceptado: 20 mayo 2021]

\begin{abstract}
Resumen: En este trabajo se han analizado las preguntas sobre alimentación y nutrición que aparecen en cinco colecciones completas de libros de texto de Educación Primaria (30 libros), prestando atención a su tipología y a cómo se distribuyen a lo largo de la etapa educativa. El estudio ha permitido identificar los tipos de preguntas que aparecen, así como su adecuación a las prescripciones curriculares de la etapa. La mayor cantidad de preguntas se concentra en los tres primeros cursos de Educación Primaria, donde también existe más diversidad de contenidos. La mayoría de las preguntas identificadas plantean demandas de tipo declarativo que implican un bajo nivel cognitivo y apenas aparecen preguntas que fomenten la descripción o la comprobación.
\end{abstract}

Palabras clave: Preguntas; Alimentación; Nutrición; Libros de texto; Educación Primaria

Food and nutritional questions do on Primary Education textbooks of Natural Sciences address

Abstract: In this work the questions about food and nutritional that appeared in five complete collections of Primary Education textbooks (30 textbooks) have been analysed, paying attention to their typology and how they are distributed throughout the educational stage. The study has allowed to identify the types of questions that appear, as well as their adaptation to the curricular prescriptions of the stage. The largest number of questions is concentrated in the first three years of Primary Education, where there is more diversity of contents. Most of the questions identified pose declarative demands that imply a low cognitive level and there are hardly any questions that encourage description or verification.

Keywords: Questions; Food; Nutrition; Textbooks; Primary Education

Para citar este artículo: Gil González C., Cortés Gracia A.L. (2021) Preguntas sobre alimentación y nutrición planteadas en los libros de texto de Ciencias de la Naturaleza en Educación Primaria. Revista Eureka sobre Enseñanza y Divulgación de las Ciencias 18(3), 3104. doi: 10.25267/Rev_Eureka_ensen_divulg_cienc.2021.v18.i3.3104

\section{Introducción}

La formulación de preguntas ha llevado al descubrimiento de las aportaciones científicas más relevantes. De hecho, se puede afirmar que la capacidad de los humanos de hacer preguntas, imaginar y buscar las respuestas está en el origen de la cultura y en concreto del conocimiento científico (Wartosfsky 1968). De esta manera, en el proceso de enseñanza y aprendizaje las preguntas tienen un papel fundamental, ya que permiten establecer relaciones entre los hechos o fenómenos objeto de estudio, el propio conocimiento y el conocimiento científico (Roca et al. 2013).

Existe consenso en la comunidad educativa sobre la importancia de las preguntas como promotoras del aprendizaje activo y la construcción del conocimiento, tanto a nivel personal como social (Sanmartí y Márquez 2012). El planteamiento de preguntas permite al alumnado avanzar en su propia comprensión y, como tal, representa una poderosa actividad metacognitiva. Sin embargo, el profesorado tiene dificultades muchas veces para formular preguntas de calidad y tiende a priorizar aquellas que demandan respuestas cortas, reproductivas y con tendencia memorística (Eslava y Eslava 2000; Márquez y Roca 2006; Miralles et al. 2014; Polanco 2004). Su preocupación por transmitir las ideas sin errores y/o el

\footnotetext{
Revista Eureka sobre Enseñanza y Divulgación de las Ciencias

Universidad de Cádir. APAC-Eureka. ISSN: 1697-011X

bttp://doi.org/10.25267/Rev_Eureka_ensen_divulg_cienc.2021.v18.i3.3104

http:/ / reuredc.uca.es
} 
temor a no saber dar una respuesta clara y completa, hace que el profesorado enfatice en preguntas dirigidas y cerradas. Incluso el propio sistema educativo o los libros de texto llegan a burocratizar las preguntas (Freire y Faundez 2013), estableciendo los tipos y momentos en los que se realizan o acompañándolas de las respuestas (es decir, son respuestas antes que preguntas). De esta forma se dejarían a un lado las preguntas abiertas, aquellas con las que se motiva al alumnado a buscar información, a reelaborar sus ideas y que promueven respuestas más complejas tanto desde un punto de vista cognitivo como lingüístico (Benedict-Chambers et al. 2017; Biggers 2018; De Rivera et al. 2005; Wells 2002). Freire y Faundez (2013) discuten esta situación y plantean cuestiones muy interesantes sobre la pedagogía de la pregunta y la necesidad de aprender a preguntar: «(Faundez) Tengo la impresión (y no sé si estás de acuerdo conmigo) de que hoy la enseñanza, el saber, es respuesta y no pregunta. (Freire)... y ni siquiera existe una demanda: jel educador, en general, ya trae la respuesta sin que le hayan preguntado nada! (Faundez)... Exactamente. Y lo más grave es que el estudiante se acostumbra a ese tipo de trabajo. Entonces, ante todo el profesor debería enseñar - porque él mismo debería saberlo - a preguntar. Porque el inicio del conocimiento, repito, es preguntan» (p. 69).

Como se ha comentado, buena parte del conocimiento que se llega a adquirir depende del tipo y la calidad de las preguntas que aparecen a lo largo de la escolarización (interacción en el aula, recursos didácticos). Teniendo en cuenta la importancia que tienen los contenidos sobre alimentación y nutrición en el currículo de Educación Primaria, así como en el desarrollo de competencias básicas y la alfabetización científica en general, parecen necesarias investigaciones que muestren el uso que se hace de las preguntas en los libros de texto a la hora de tratar contenidos sobre aspectos de alimentación y nutrición.

\section{Marco teórico}

\section{Tipos de preguntas y demandas cognitivas}

Abrandt-Dahlgren y Öberg (2001) plantean una categorización de las preguntas que recoge cinco tipos diferentes: enciclopédicas, de comprensión, relacionales, de evaluación y de búsqueda de soluciones. A partir de esta categorización, autoras como Leite et al. (2012) y Martins et al. (2014) señalan que las preguntas enciclopédicas implicarían un nivel cognitivo inferior al resto ya que no requieren necesariamente reflexión o comprensión de los temas. Del mismo modo, también indican que con un enfoque de la enseñanza basado en «... una metodología tradicional, apoyada en la lectura del manual, (...) podemos encontrar una justificación plausible de la dificultad de los estudiantes para plantear cuestiones de un nivel cognitivo más exigente. Por lo tanto, se entiende que, de acuerdo con otros estudios, a los estudiantes no se les enseñó a cuestionar» (Martins et al. 2014, p. 255).

Roca et al. (2013) distinguen entre el presupuesto de la pregunta (que hace referencia a un campo de conocimiento) y la demanda de la misma (que hace referencia al objetivo de quien la plantea). En el trabajo citado, las autoras proponen un conjunto de categorías de análisis para las preguntas del alumnado en función del objetivo o demanda de las mismas (tabla 1).

Tabla 1. Categorías de análisis del objetivo o demanda de la pregunta según Roca et al. (2013).

\begin{tabular}{|c|}
\hline Descripción \\
\hline Explicación causal \\
\hline Comprobación \\
\hline Generalización, definición \\
\hline Predicción \\
\hline Gestión \\
\hline Evaluación, opinión \\
\hline
\end{tabular}


Por lo tanto, ya que son una de las herramientas pedagógicas más frecuentemente utilizadas en las aulas y verdaderas activadoras del conocimiento científico (Colás 1983; Montenegro 2002), parece necesaria una formación docente que muestre al profesorado el impacto que tiene una buena formulación de preguntas en el proceso de enseñanza y aprendizaje y, al mismo tiempo, se le indique la importancia que tienen en su diseño aspectos como el contexto, el nivel escolar y la coherencia las mismas (González 2007; Polanco 2004; Roca 2007; Tornero et al. 2015). Lo anterior también debería extenderse a poner en valor la importancia que tienen las preguntas planteadas por el alumnado, especialmente por su relación con la construcción del conocimiento y el desarrollo del pensamiento crítico (Chin 2001; Chin y Osborne 2008; Roca et al. 2013). Aunque sus preguntas suelen ser generales, pobres y cerradas, en un ambiente de clase adecuado el alumnado es capaz de plantear desde las preguntas más simples hasta las que manifiestan un pensamiento profundo complejo, lo cual permite al profesorado detectar su nivel o capacidad de comprensión conceptual (Alsop et al. 2002; Woodward 1992). Igualmente, puesto que los recursos educativos empleados por el profesorado son mediadores clave del proceso de enseñanza y aprendizaje, las preguntas planteadas en ellos suscitan un gran interés para la investigación educativa.

\section{La importancia de los libros de texto}

En el marco de la enseñanza y aprendizaje de las Ciencias de la Naturaleza, las preguntas tienen mucho protagonismo al estar presentes en todo tipo de actividades, desde las intervenciones del profesorado y alumnado en el día a día hasta los deberes escolares y exámenes. Por ello hay que prestar atención especialmente a las que aparecen en el libro de texto por ser el material educativo más utilizado en el aula y convertirse, en bastantes ocasiones, en el referente exclusivo del currículo escolar y del saber científico (Braga y Belver 2016; Harrison 2001; López-Valentín y Guerra-Ramos 2012; Martínez y Rodríguez 2010; Torres-García et al. 2018). Como principal material didáctico en muchos casos, el libro de texto ejerce una notable influencia en el aprendizaje del alumnado al ser la fuente de información de referencia y dirigir muchas de sus tareas. Distintas investigaciones muestran desde hace años que el conocimiento que se construye en las aulas a través de estrategias tradicionales como el uso exclusivo de los libros de texto se encuentra, en muchas ocasiones, alejado de lo que debería ser una adecuada alfabetización científica (Perales y Vílchez 2012; Pozuelos et al. 2016; Pujol et al. 2006). Muchos de los contenidos se presentan como simples etiquetas que se deben memorizar sin conexiones con el resto de elementos de los sistemas que intervienen (Ben-Zvi et al. 2010; Riess y Mischo 2010) o simplemente no se evalúan (Rivadulla et al. 2017). En el caso de la visión sistémica del modelo de ser vivo, alimentación y nutrición se usan indistintamente y pocas veces se aborda una perspectiva general en la que se conecten, por ejemplo, respiración, circulación, nutrición y medio.

\section{Los contenidos sobre alimentación y nutrición en Educación Primaria}

Los conocimientos sobre alimentación y nutrición suscitan un gran interés tanto a nivel educativo como en la vida cotidiana. Por ejemplo, el sistema educativo español, el bloque 2 del currículo de Educación Primaria de la asignatura troncal Ciencias de la Naturaleza (LOMCE 2013) establece, entre los contenidos mínimos, la función de nutrición y los hábitos saludables, entre otros. La importancia de la alimentación y la nutrición se pone de manifiesto asimismo en el interés del tema en la investigación educativa. Así, existen numerosos trabajos centrados en temas concretos de las etapas de Educación Infantil y Primaria (Cubero et al. 2012 2017, Núñez y Banet 2000; Rivadulla et al. 2017; Rivadulla et al. 2020), de Educación Secundaria Obligatoria (Bizzio et al. 2015; Cabello et al. 2016) e incluso en la formación del profesorado (García et al. 2010). El desarrollo de la competencia en alimentación es objeto de diversos trabajos como los de España et al. (2014) o Cabello et al. (2016). Los niveles competenciales 
presentes en los contenidos sobre alimentación de los libros de texto son analizados por Garzón et al. (2018), Rodríguez et al. (2018) o Torres-García et al. (2018).

\section{Objetivos}

Teniendo en cuenta lo anterior, el objetivo principal de este trabajo es identificar los tipos de preguntas sobre alimentación y nutrición que aparecen en los libros de texto de Ciencias de la Naturaleza en Educación Primaria de acuerdo con su demanda cognitiva, así como su distribución a lo largo de toda la etapa educativa.

\section{Metodología}

Se ha realizado un análisis de contenido sobre una muestra de cinco colecciones completas (30 libros, ver Anexo I) del periodo 2014-2018 de libros de texto de Ciencias de la Naturaleza de Educación Primaria (en adelante EP). Las colecciones analizadas corresponden a varias de las editoriales más vendidas en España, entendiendo que pueden ser una muestra representativa de los libros de texto españoles (tabla 2). El análisis se centra especialmente en las unidades temáticas donde deberían aparecer estos contenidos de acuerdo con el currículo oficial (LOMCE 2013).

Tabla 2. Nomenclatura utilizada para cada una de las editoriales analizadas. Elaboración propia.

\begin{tabular}{|c|c|c|}
\hline NOMENCLATURA Y COLOR & EDITORIAL \\
\hline Editorial 1 & & Anaya \\
\hline Editorial 2 & & Edebé \\
\hline Editorial 3 & & Edelvives \\
\hline Editorial 4 & & Santillana \\
\hline Editorial 5 & & Vicens Vives \\
\hline
\end{tabular}

En palabras de López-Noguero (2002), el análisis de contenido «sólo puede entenderse de una forma completa si lo situamos en el contexto de un diseño de investigación». Así, la definición de las unidades de análisis y categorías es uno de sus principales problemas y hace que no existan plantillas de análisis generalizables al depender de los objetivos de la investigación (Occelli y Valeiras 2013).

De esta manera, antes de realizar el análisis se ha elaborado un sistema de categorías para las preguntas que pueden aparecer en los libros de texto usando como referencia las señaladas por Roca et al. (2013) para el análisis del objetivo o demanda de las preguntas de los alumnos. Al listado de categorías propuesto por estas autoras se han añadido otras dos. La categoría "declaración" haría referencia a preguntas que demandan una respuesta directa y determinada. Es decir, su objetivo es obtener información o afirmación sobre un hecho concreto mediante una reproducción declarativa del conocimiento (por ejemplo, ¿desayunas antes de salir de casa?). La categoría "introducción" tiene que ver con una característica habitual de muchos libros de texto, como es la presencia de preguntas explícitas en la introducción o presentación del tema antes de dar paso a los contenidos principales del mismo. Por ello, la forma de la pregunta puede ser similar a la de otras categorías, aunque la intención didáctica de los autores o el uso que hagan de ella profesorado y alumnado pueden no coincidir (por ejemplo, junto a una fotografía en la que aparecen numerosos tipos de frutas y verduras se pregunta ¿qué fruta elegirías para la merienda?). Posteriormente, las categorías recopiladas se han incluido en una plantilla de análisis de elaboración propia (tabla 3) que se ha aplicado a la muestra completa de libros de texto. 
Tabla 3. Plantilla de análisis de elaboración propia empleada para la recopilación de las preguntas sobre alimentación y nutrición que aparecen en los libros de texto estudiados (*en los libros analizados no se han identificado preguntas de comprobación). Elaboración propia.

\begin{tabular}{|c|c|c|}
\hline \multicolumn{3}{|c|}{ TIPOS DE PREGUNTAS } \\
\hline Categoría & Forma de la pregunta & Ejemplos en los libros analizados \\
\hline Descripción & $\begin{array}{c}\text { ¿Cómo? ¿Dónde? ¿Quién? ¿Cuántos? ¿Qué pasa? } \\
\text { ¿Cómo pasa? }\end{array}$ & $\begin{array}{c}\text { ¿Cómo la vais a decorar, haciendo formas } \\
\text { con las frutas, añadiendo zumo de naranja o } \\
\text { yogur...? }\end{array}$ \\
\hline Explicación causal & $\begin{array}{c}\text { ¿Por qué? ¿Cuál es la causa? } \\
\text { ¿Cómo es que? ¿A causa de qué? }\end{array}$ & ¿Por qué hay que comer de forma saludable? \\
\hline Comprobación* & $\begin{array}{l}\text { ¿Cómo se puede saber? ¿Cómo lo saben? ¿Se } \\
\text { puede demostrar que? ¿Son posibles los } \\
\text { resultados en la prueba? ¿Cómo se hace? }\end{array}$ & $\begin{array}{l}\text { ¿Cómo se puede saber si un alimento } \\
\text { contiene azúcar? (ejemplo propio)* }\end{array}$ \\
\hline Generalización/definición & $\begin{array}{l}\text { ¿Qué es? (Definición) ¿Pertenece a tal grupo? } \\
\text { ¿Qué diferencia hay? ¿Por qué según la teoría? }\end{array}$ & ¿Qué es la salud? \\
\hline Predicción & $\begin{array}{c}\text { ¿Qué consecuencias? ¿Qué puede pasar? ¿Podría } \\
\text { ser? ¿Qué pasará sí...? Formas verbales de } \\
\text { futuro o condicionales. }\end{array}$ & $\begin{array}{c}\text { ¿Qué harías si te sobrara un trozo de } \\
\text { bocadillo? }\end{array}$ \\
\hline Declaración & $\begin{array}{c}\text { ¿Cuánto es más...que? ¿En qué fecha...? ¿Cuál } \\
\text { es...? ¿ Cuántas...? }\end{array}$ & $\begin{array}{l}\text { ¿Cuáles de estos alimentos son de origen } \\
\text { animal? }\end{array}$ \\
\hline Introducción & $\begin{array}{l}\text { ¿Cómo? ¿Dónde? ¿Qué? ¿Cuántos? ¿Qué pasa? } \\
\text { ¿Cómo ocurre? ¿Por qué? ¿Debido qué? ¿Cómo } \\
\text { es que...? ¿Cómo se puede saber...? ¿Cómo lo } \\
\text { saben? ¿Cómo se hace? ¿Qué se puede deducir } \\
\text { de los datos? ¿Por qué se toman estas medidas y } \\
\text { no otras? ¿Por qué se comparan estos datos? }\end{array}$ & ¿Qué alimentos reconoces en esta imagen? \\
\hline
\end{tabular}

Una vez establecidas todas las categorías, se ha realizado el análisis de contenido con el que se han extraído y clasificado todas las preguntas planteadas por las distintas editoriales en cada curso. En general, los contenidos se encuentran incluidos en unidades temáticas específicas sobre la alimentación y la función de nutrición (normalmente una unidad en cada curso), tanto en la parte correspondiente al texto principal como a las actividades. Como unidad básica de análisis se ha considerado cada una de las preguntas individuales que se formulan de forma explícita en los libros.

Los resultados obtenidos han sido cuantificados para poder conocer la cantidad de preguntas planteadas según las editoriales analizadas. Se ha diferenciado por curso académico y categoría para cada uno de ellos. Hay que tener en cuenta que el número total de preguntas es un dato orientativo y coyuntural ya que depende claramente de la existencia de contenidos sobre alimentación y nutrición en cada uno de esos libros. En un estudio reciente, Gil y Cortés (2020) señalan que la mayor parte de los contenidos sobre alimentación y nutrición se concentran en los tres primeros cursos de EP, especialmente en los libros de tercer curso, y este hecho es coincidente en las principales editoriales españolas, lo que podría condicionar claramente la existencia o no de preguntas sobre la temática estudiada en los libros de las colecciones analizadas.

\section{Resultados}

El análisis de contenido ha permitido identificar un total de 361 preguntas explícitas que han aparecido en las cinco colecciones completas de libros de texto. La colección que más preguntas plantea entre todos los cursos es la correspondiente a la editorial 4 , con un total de 91 preguntas, mientras que la editorial 1, con un total de 52 preguntas, es la que menos preguntas explícitas plantea. 


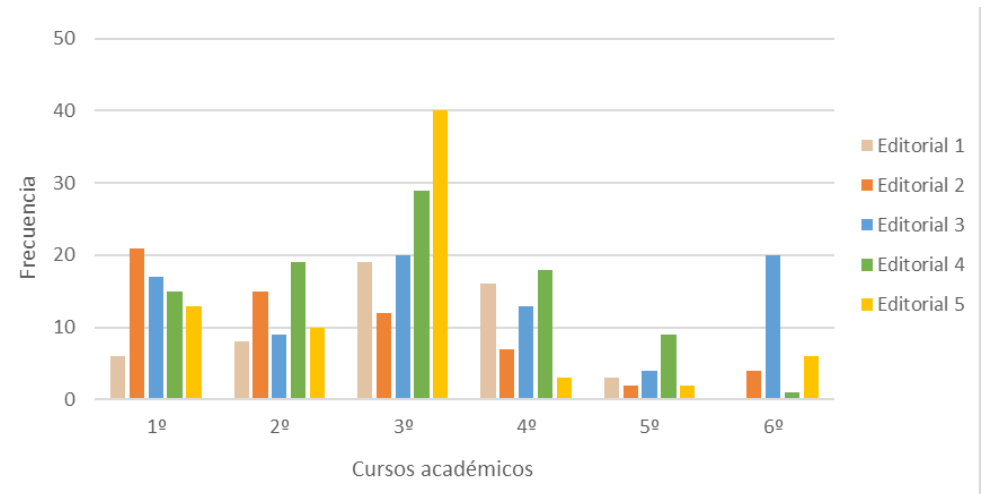

Figura 1. Número de preguntas planteadas por las editoriales en cada curso académico. Elaboración propia.

En los tres primeros cursos de la etapa (figura 1), especialmente en $3^{\circ} \mathrm{EP}$, aparece el mayor número de preguntas sobre alimentación y nutrición (un total de 120 preguntas), de forma coherente a la gran cantidad y diversidad de contenidos relacionados que muestran los libros de texto de estos niveles tal y como indicaban Gil y Cortés (2020). Las editoriales 4 y 5 son las que más preguntas plantean con un total de 63 preguntas cada una de ellas, mientras que la editorial 1 , especialmente en $1^{\circ}$ y $2^{\circ} \mathrm{EP}$, es la que menor número de preguntas muestra en el conjunto de estos tres cursos. Hay que destacar el elevado número de preguntas planteadas en el libro de $3^{\circ}$ de la editorial 5 , con el máximo absoluto en todo el estudio $(\mathrm{n}=40)$. De la misma manera, debido a la escasa aparición de estos contenidos en los tres cursos restantes, el número de preguntas que incluyen cada una de las editoriales se ve claramente reducido, especialmente en $5^{\circ} \mathrm{EP}$, e incluso desaparecen, como es el caso de la editorial 1 en $6^{\circ} \mathrm{EP}$. En este caso, también destaca el elevado número de preguntas planteadas por la editorial 3 en $6^{\circ}$ EP.

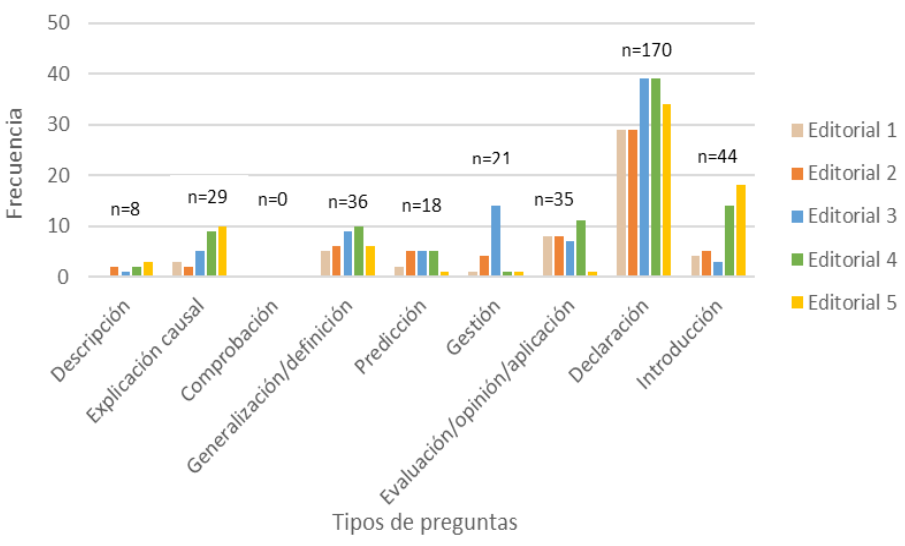

Figura 2. Número de preguntas por categorías en las editoriales estudiadas. Elaboración propia.

En cuanto a los tipos de preguntas que aparecen en las editoriales estudiadas (figura 2), las que buscan respuestas declarativas (categoría "declaración") son las más frecuentes en todas ellas con un total de 170 preguntas. Por el contrario, la categoría "comprobación” está ausente en todas las editoriales y la categoría "descripción" es la que menos se plantea (tan solo 8 preguntas) llegando a estar ausente en la editorial 1. 


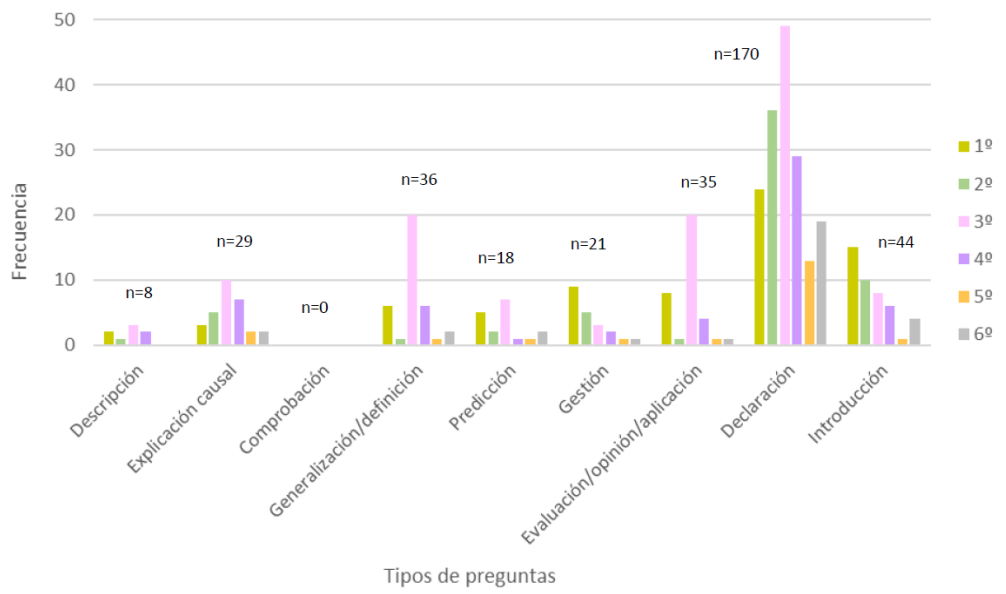

Figura 3. Número de preguntas por categorías en cada curso. Elaboración propia.

Respecto a los tipos de preguntas que aparecen en cada curso (figura 3), vuelve a destacar la categoría "declaración" por englobar el mayor número de preguntas, especialmente en $3^{\circ} \mathrm{EP}$. Esto implica una demanda cognitiva muy escasa, ya que el objetivo se reduce a obtener respuestas directas y poco elaboradas. Además, de forma paralela a la reducción de contenidos sobre alimentación y nutrición que muestran los libros de texto en los cursos posteriores, las preguntas de "gestión" e "introducción" se reducen gradualmente a lo largo de toda la etapa y las que demandan una "descripción" son muy escasas e incluso están ausentes en $5^{\circ}$ y $6^{\circ}$ EP. Por otro lado, las categorías "explicación causal" y "evaluación, opinión, aplicación", que desde una perspectiva teórica podrían relacionarse con niveles posteriores del desarrollo cognitivo del alumnado, alcanzan su máximo en $3^{\circ} \mathrm{EP}$ y se reducen claramente en los cursos posteriores.

A pesar de la cantidad y variedad de preguntas planteadas por todas las editoriales, algunas se repiten en el mismo curso o en varios cursos. Este es el caso de preguntas como iqué sueles desayunar?, formulada en $2^{\circ}$ EP como actividad por las editoriales 3 y 4 ; ¿qué comemos?, planteada como título de un apartado teórico en $1^{\circ} \mathrm{EP}$ por la editorial 1 , en $1^{\circ}$ y $2^{\circ} \mathrm{EP}$ por la editorial 3 y en $3^{\circ} \mathrm{EP}$ por la editorial 5; ¿por qué crees que el desayuno es una comida muy importante?, planteada como actividad por la editorial 4 en $4^{\circ}$ y $5^{\circ} \mathrm{EP}$; ¿qué es la salud?, planteada como título de un apartado teórico en $3^{\circ}$ y $4^{\circ}$ EP por las editoriales 3 y 4 respectivamente y como actividad en $5^{\circ} \mathrm{EP}$ por la editorial 2; y ies lo mismo alimento que nutriente?, planteada como actividad en $3^{\circ}$ y $5^{\circ} \mathrm{EP}$ por las editoriales 3 y 4 respectivamente.

Finalmente, hay que señalar la existencia de numerosas preguntas acerca de la importancia del desayuno, aunque no necesariamente son formuladas por todas las editoriales y en los mismos cursos. Por ejemplo, en $3^{\circ} \mathrm{EP}$ son las editoriales 2 y 5 las que formulan estas preguntas mientras que en el resto de cursos lo hacen las editoriales 3 y 4 .

\section{Discusión y conclusiones}

El análisis realizado ha permitido identificar hasta 361 preguntas explícitas sobre alimentación y nutrición en los contenidos de las cinco colecciones completas de los libros de texto de Ciencias de la Naturaleza de Educación Primaria del periodo 2014-2018.

La cantidad y distribución de contenidos sobre alimentación y nutrición que aparecen en los libros de texto indicada en el trabajo de Gil y Cortés (2020) nos permite señalar que es coherente que el número de preguntas que muestran cada una de las editoriales sufra un aumento o reducción conforme avanzan los cursos académicos, ya que coincide con la variación general de contenidos sobre el tema a lo largo de la etapa. Esta distribución de los 
contenidos no responde exactamente a las prescripciones curriculares (LOMCE 2013) sino más bien a criterios editoriales. Como también indican Pozuelos et al. (2016), basándose en diversos trabajos previos, los libros de texto no solo recogen la interpretación del currículum oficial, sino que se llegan a convertir en el currículum oficial en muchos casos. Perales y Vílchez (2012, p. 77) añaden que "normalmente son las editoriales las que imponen la extensión del libro, y los editores los que se encargan de encajar la idea de los autores en el espacio disponible". Así, en los tres primeros cursos y, especialmente en $3^{\circ} \mathrm{EP}$, aparece el mayor número de preguntas debido a la gran cantidad y diversidad de contenidos sobre alimentación y nutrición que abordan los libros de texto en estos cursos y que es coincidente en todas las editoriales. A pesar de existir bastantes preguntas en esos cursos, el predominio de demandas que buscan simples declaraciones o generalizaciones tienen que ver con una imagen de ciencia afirmativa (Roca et al. 2013), alejada de la búsqueda de pruebas y el desarrollo del pensamiento crítico. En los siguientes cursos, como consecuencia de los escasos contenidos sobre alimentación y nutrición que muestran los libros de texto analizados (especialmente en $\left.5^{\circ} \mathrm{EP}\right)$ el número de preguntas planteadas también sufre un acusado descenso y el tipo de demanda es equivalente, con un dominio del tipo declarativo en busca de respuestas directas. Quizás la escasez de contenidos sobre alimentación y nutrición, así como buenas preguntas sobre ello en $5^{\circ}$ y $6^{\circ}$, sea una oportunidad perdida si tenemos en cuenta el énfasis que muchos autores hacen sobre el desarrollo de la competencia en alimentación en el periodo de 10 a 17 años (Cabello et al. 2016; Del Carmen 2010, entre otros).

De la misma manera, comparando los resultados de este estudio con los contenidos sobre alimentación y nutrición prescritos en el currículo de EP (LOMCE 2013) y en sus desarrollos autonómicos, se observa que esos contenidos curriculares no siempre son abordados en las preguntas que aparecen en los cursos indicados y que, además, muchos de los contenidos que sí aparecen demandados a través de preguntas surgen en cursos a los que no corresponden de acuerdo al currículo de EP. Por ejemplo, los contenidos sobre alimentación y nutrición que prescribe el currículo en los tres primeros cursos de la etapa (fundamentalmente relacionados con el conocimiento de prácticas y hábitos saludables) apenas se ven reflejados en las preguntas planteadas por ninguna de las editoriales. De esta forma, como se comentaba anteriormente, se pierde la oportunidad de que las niñas y niños expresen sus conocimientos y sus modelos respecto a los contenidos planteados para esos niveles cognitivos. Sin embargo, aparecen contenidos y preguntas correspondientes teóricamente a cursos posteriores como los relacionados con el menú saludable, el etiquetado, los nutrientes o la rueda de los alimentos, entre otros. Esta situación se repite en el resto de cursos, a pesar de la amplia variedad de contenidos sobre alimentación y nutrición que se podrían o deberían tratar y cuyo aprendizaje se podría facilitar mediante el planteamiento de "buenas preguntas". De hecho, contenidos específicos para estos cursos como la dieta mediterránea, la conservación de alimentos y la potabilización del agua, muy interesantes desde el punto de vista de la alfabetización científica, no son abordados a través de preguntas en ninguno de estos niveles, y, sin embargo, en $6^{\circ}$ de Primaria las preguntas que aparecen tan solo hacen referencia a la dieta y los hábitos saludables, y en general son demandas de tipo declarativo. De nuevo se pierde la oportunidad de plantear demandas de mayor nivel cognitivo a través de preguntas de opinión, explicación o comprobación.

En cuanto al tipo de preguntas que formulan las distintas editoriales, autores como Odom y Bell (2011) afirman que las que corresponden a la categoría "declaración" apenas favorecen la construcción del conocimiento y que, además, para responderlas tan solo se requieren bajas habilidades de pensamiento, al tener que recordar únicamente términos o ideas establecidas. Por tanto, resulta llamativo el elevado número de preguntas de esta categoría que aparecen planteadas en los libros de texto (un total de 141 preguntas). Sin embargo, preguntas que 
conducen al conocimiento científico, al desarrollo del pensamiento crítico, al planteamiento de nuevas preguntas, a la necesidad de indagar en busca de pruebas o incluso experimentar (Benedict-Chambers et al. 2017; Jiménez-Liso 2020; Roca 2007) apenas son planteadas por las editoriales, llegando incluso a estar ausentes en toda la etapa, como ocurre con la categoría "comprobación". Este resultado sería contradictorio con el concepto actual que tenemos de la competencia científica (Sanmartí y Márquez 2012), la cual según la OCDE-PISA (2006) hace referencia a la capacidad de identificar preguntas que puedan ser investigadas científicamente en una situación dada y de detectar los términos clave para buscar información científica sobre un determinado tema. A pesar de los esfuerzos que desde la investigación en Didáctica de las Ciencias Experimentales se están haciendo en las últimas décadas por indicar la necesidad de un aprendizaje de las ciencias basado en la argumentación, el uso de pruebas y la modelización para la explicación-predicción de fenómenos (Couso et al. 2020; Jiménez-Aleixandre 2010; Jiménez-Liso et al. 2019), los libros de texto de Educación Primaria no recogen ese enfoque. En este sentido, Perales y Vílchez (2012) señalaban que las editoriales suelen imponer la lógica de la disciplina correspondiente y apenas dan oportunidades para incluir innovaciones y enfoques procedentes de la investigación educativa. Así siguen mostrando un conjunto de contenidos cerrados y presentan abundantes preguntas con demandas meramente declarativas que probablemente, como indicaban Freire y Faundez (2013), se plantearon por los autores como respuestas y no como preguntas.

Por otro lado, los resultados del análisis ponen de manifiesto el gran interés que ha suscitado el desayuno saludable en nuestra sociedad desde hace años, que supuso la puesta en marcha de numerosos proyectos escolares y sanitarios tanto a nivel nacional como autonómico (Banet y López 2010; Cubero et al. 2013 2017; Segovia y Villares 2010, entre otros). Así queda recogido en los textos de las distintas editoriales analizadas, siendo abordado por varias de ellas con contenidos y preguntas explícitas a lo largo de toda la etapa. Frente a este hecho, que indica que las editoriales sí parecen recoger algunas de las recomendaciones para la alfabetización y el desarrollo de la competencia en alimentación procedentes de la comunidad científica, encontramos que no se abordan otras cuestiones como la publicidad alimentaria o la influencia de los medios de comunicación, muy interesantes desde el punto de vista del desarrollo de destrezas argumentativas y que tienen gran influencia en la educación alimentaria y en la salud (Goris 2009; Royo y Rodríguez 2015).

En conclusión, dada la importancia que tienen los libros de texto en nuestro sistema educativo y que el tipo y la calidad de las preguntas formuladas determina muchos de los conocimientos que el alumnado llega a adquirir, parece necesario revisar y repensar tanto la distribución de contenidos como las preguntas que se formulan en los mismos sobre alimentación y nutrición para garantizar una Educación Alimentaria efectiva y que permita desarrollar el pensamiento crítico frente a la influencia ejercida por la sociedad y los medios de comunicación en el ámbito de la salud y la alimentación.

\section{Agradecimientos}

A los revisores, por sus acertados comentarios y sugerencias. Grupo de referencia BEAGLE Investigación en Didáctica de Ciencias Naturales (Gobierno de Aragón y Fondo Social Europeo) Instituto de Investigación en Ciencias Ambientales de Aragón (IUCA/UNIZAR). Proyecto EDU2016-76743-P (MINECO). Cristina Gil González disfruta de un contrato predoctoral del Gobierno de Aragón (ORDEN IIU/1408/2018), Programa Operativo FSE Aragón 2014-2020, Construyendo Europa desde Aragón.

\section{Referencias}

Abrandt-Dahlgren M., Öberg G. (2001) Questioning to learn and learning to question: Structure and function of problem-based learning scenarios in environmental science education. Higher Education, 41, 263-282. https://doi.org/10.1023/A:1004138810465 
Alsop S., Gould G., Watts M. (2002) The role of pupils' questions in learning science. En S. Amos, R. Booham (eds), Aspects of teaching secundary science (pp. 39-48). London: The Open University.

Banet E., López C. (2010) ¿Cómo mejorar el desayuno de los escolares de educación primaria? Investigación en la escuela, 71, 63-83. https://doi.org/10.12795/IE.2010.i71.06

Ben-Zvi O., Orion N. (2010) System Thinking Skills at the Elementary School Level. Journal of Research in Science Teaching, 47(5), 540-563. https://doi.org/10.1002/tea.20351

Benedict-Chambers A., Kademian S. M., Davis E. A., Sullivan-Palincsar A. (2017) Guiding students towards sensemaking: teacher questions focused on integrating scientific practices with science content. International Journal of Science Education, 39(15), 1977-2001. https://doi.org/10.1080/09500693.2017.1366674

Biggers M. (2018) Questioning Questions: Elementary Teachers' Adaptations of Investigation Questions Across the Inquiry Continuum. Research in Science Education, 48, 1-28. https://doi.org/10.1007/s11165-016-9556-4

Bizzio M. A., Pereira R. A., Nuñez G. I., Morales L. M. (2015) Aprendiendo a seleccionar alimentos en el recreo escolar. Alambique Didáctica de las Ciencias Experimentales, 79, 73-78.

Braga G., Belver J. L. (2016) El análisis de libros de texto: una estrategia metodológica en la formación de los profesionales de la educación. Revista Complutense de Educación, 27(1), 199-218. http://dx.doi.org/10.5209/rev_RCED.2016.v27.n1.45688

Cabello A., España E., Blanco A. (2016) La competencia en alimentación. Barcelona: Octaedro.

Chin C. (2001) Learning in Science: What Do Students' Questions Tell Us About Their Thinking? Education Journal, 29(2), 85-103. http://hdl.handle.net/10497/4734

Chin C., Osborne J. (2008) Students' questions: a potential resource for teaching and learning science. Studies in Science Education, 44(1), 1-39. https://doi.org/10.1080/03057260701828101

Colás M. P. (1983) La formulación de preguntas en el acto didáctico: un estudio comparativo. Enseñanza \& Teaching: Revista Interuniversitaria de didáctica, 1, 77-86.

Couso D., Jiménez-Liso M. R., Refojo C., Sacristán J. A. (Coords) (2020) Enseñando Ciencia con Ciencia. FECYT \& Fundacion Lilly. Madrid: Penguin Random House.

Cubero J., Cañada F., Costillo E., Franco L., Calderón A., Santos A. L., Pádez C., Ruiz C. (2012) La alimentación preescolar, educación para la salud de los 2 a los 6 años. Enfermería Global, 11(3), 337-345. https://doi.org/10.6018/eglobal.11.3.139041

Cubero J., Franco-Reynolds L., Calderón M. A., Caro B., Rodrigo M., Ruiz C. (2017) El desayuno escolar; una intervención educativa en alimentación y nutrición saludable. Didáctica de las Ciencias Experimentales y Sociales, 32(1), 171-182. https://doi.org/10.7203/DCES.32.4546

Cubero J., Guerra M., Costillo Borrego E., Pozo E., Ruiz Macías C. (2013) Análisis del desayuno en una población de escolares del $3^{\circ}$ ciclo de Primaria; un recurso didáctico en Educación para la Salud. Campo abierto: Revista de educación, 32(2), 145-153.

De Rivera C., Girolametto L., Greenberg J., Weitzman E. (2005) Children's responses to educators' questions in day care play groups. American Journal of Speech-Language Pathology, 14, 14-26. https://doi.org/10.1044/1058-0360(2005/004) 
Eslava de Aja L., Eslava J. (2000) La pregunta oral y escrita como factor de interacción maestro-alumno en el aula. Revista de educación en Ciencias, 1(2), 81-86.

España E., Cabello A., Blanco A. (2014) La competencia en alimentación. Un marco de referencia para la educación obligatoria. Enseñanza de las Ciencias, 32 (3), 611-129.

Freire P., Faundez A. (2013) Por una pedagogía de la pregunta: crítica a una educación basada en respuestas a preguntas inexistentes. Buenos Aires: Siglo Veintiuno Editores.

García S., Martínez C., Rivadulla J. C. (2010) La percepción medioambiental del profesorado de primaria en el tema de la nutrición humana. Revista Eureka sobre enseñanza y divulgación de la ciencia, 7, 286-296. http://dx.doi.org/10.25267/Rev_Eureka_ensen_divulg_cienc.2010.v7.iextra.11

Garzón A., Talavera M., Gavidia V. (2018) Niveles de competencia en alimentación y actividad física en los libros de texto de Educación Secundaria Obligatoria. Didáctica de las Ciencias Experimentales y Sociales, 36, 61-78. https://doi.org/10.7203/DCES.36.12186

Gil C., Cortés A. L. (2020) ¿Qué contenidos sobre alimentación abordan los libros de texto de Ciencias de la Naturaleza en Educación Primaria? Ápice. Revista de Educación Científica, 4(2), 17-33. https://doi.org/10.17979/arec.2020.4.2.6529

González, J. (2007) Debates en el aula preescolar a partir de cuentos: Estudio comparado en España y México. Perfiles educativos, 29(118), 54-78.

Goris J. M. (2009) Television food advertising and the prevalence of childhood overweight and obesity: a multicountry comparison. Public Health Nutrition, 13(7), 1003-1012. https://doi.org/10.1017/S1368980009992850

Harrison A. G. (2001) How do Teachers and Textbook Writers Model Scientific Ideas for Students? Research in Science Education, 31, 401-435. https://doi.org/10.1023/A:1013120312331

Jiménez-Aleixandre M. P. (2010) 10 Ideas clave. Competencias en argumentación y uso de pruebas. Barcelona: Graó.

Jiménez-Liso M. R. (2020) Aprender ciencia escolar implica aprender a buscar pruebas para construir conocimiento (indagación). En D. Couso, M. R. Jimenez-Liso, C. Refojo y J. A. Sacristán (coords), Enseñando Ciencia con Ciencia (pp. 53-61). FECYT \& Fundacion Lilly. Madrid: Penguin Random House.

Jiménez-Liso M. R., Giménez-Caminero E., Martínez-Chico M., Castillo-Hernández F. J., López-Gay R. (2019) El enfoque de enseñanza por indagación ayuda a diseñar secuencias: ¿Una rama es un ser vivo? En J. Solbes y M.R. Jiménez-Liso (eds.), Propuestas de Educación Científica basadas en la indagación y modelización en contexto (pp. 97-120). Valencia: Tirant lo blanch.

Leite L., Dourado L., Morgado S., Vilaça T., Vasconcelos C., Pedrosa M. A., Afonso A. S. (2012) Questionamento em manuais escolares de Ciências: desenvolvimento e validação de uma grelha de análise. Educar em Revista, 44, 127-143. https://dx.doi.org/10.1590/S0104-40602012000200009

LOMCE (2013) Ley Orgánica 8/2013, de 9 de diciembre (BOE del 10 de diciembre), para la mejora de la calidad educativa.

López-Noguero, F. (2002) El análisis de contenido como método de investigación. XXI Revista en Educación, 4, 167-179. 
López-Valentín D. M., Guerra-Ramos M. T. (2012) Análisis de las actividades de aprendizaje incluidas en libros de texto de ciencias naturales para Educación Primaria utilizadas en México. Enseñanza de las Ciencias, 31(2), 173-191. https://doi.org/10.5565/rev/ec/v31n2.815

Martínez J., Rodríguez J. (2010) El curriculum y el libro de texto escolar. Una dialéctica siempre abierta. En J. Gimeno Sacristán (comp.), Saberes e incertidumbres sobre el currículum (pp. 246-268). Madrid: Morata.

Martins R., Torres J., Moutinho S., Santos J., Vasconcelos C. (2014) El cuestionamiento en la clase de Ciencias: Desde los libros de texto hasta la formulación de preguntas por los estudiantes. Enseñanza de las Ciencias de la Tierra, 22(3), 251-256.

Márquez C., Roca M. (2006) Plantear preguntas: un punto de partida para aprender ciencia. Revista Educación y Pedagogía, 18 (45), 63-71.

Márquez C., Roca M., Gómez A., Sardá A., Pujol R. M. (2004) La construcción de modelos explicativos complejos mediante preguntas mediadoras. Investigación en la Escuela, 53, 71 81. https:// doi.org/10.12795/IE.2004.153.05

Miralles P., Gómez C. J., Sánchez, R. (2014) Dime qué preguntas y te diré qué evalúas y enseñas. Análisis de los exámenes de ciencias sociales en tercer ciclo de Educación Primaria. Aula Abierta, 42, 83-89. https://doi.org/10.1016/j.aula.2014.05.002

Montenegro I. (2002) Preguntas cognitivas y metacognitivas en el proceso de aprendizaje. Tecné, episteme y didaxis: Revista de la Facultad de Ciencia y Tecnologia, 11, 51-62. https://doi.org/10.17227/ted.num11-5602

Núñez F., Banet E. (2000) Aprender sobre la alimentación para desarrollar hábitos y actitudes saludables en el alumnado de primaria. Aula de Innovación Educativa, 92, 9-14. http://hdl.handle.net/11162/38148

Perales F. J., Vílchez J.M. (2012) Libros de texto: ni contigo ni sin ti tienen mis males remedio. Alambique Didáctica de las Ciencias Experimentales, 70, 75-82.

Polanco A. (2004) La pregunta pedagógica en el nivel inicial. Revista electrónica Actualidades Investigativas en Educación, 4(2), 1-12. https://doi.org/10.15517/AIE.V4I2.9082

Pozuelos F. J., Morcillo V., Travé, G. H. (2016) La alimentación en los libros de texto. En P. Cañal de León, G. Travé, F. J. Pozuelosa, A. M. Criado, A. García Carmona (coords.), La enseñanza sobre el medio natural y social: investigaciones y experiencias (pp. 149-173). Sevilla: Díada.

Pujol R. M., Bonil J., Márquez C. (2006) Avanzar en la alfabetización científica: Descripción y análisis de una experiencia en torno al estudio del cuerpo humano en Educación Primaria. Investigación en la Escuela, 60, 37-52. https://doi.org/10.12795/E.2006.i60.03

Riess W., Mischo C. (2010) Promoting Systems Thinking through Biology Lessons. International Journal of Science Education, 32(6), 705-725. https://doi.org/10.1080/09500690902769946

Rivadulla J. C., García S., Fuentes M. J., Golías Y. (2020) Los hábitos alimenticios del alumnado de Primaria y sus posibilidades educativas. Ápice. Revista de Educación Cientifica, 4(1), 6378. https://doi.org/10.17979/arec.2020.4.1.5236

Rivadulla J., García S., Martínez C. (2017) Qué enseña y evalúa sobre nutrición humana un grupo de profesores españoles y portugueses de educación primaria. Enseñanza de las Ciencias, 35(3), 69-87. https://doi.org/10.5565/rev/ensciencias.2347 
Roca M. (2007) Les preguntes en l'aprenentatge de les ciències. Tesis doctoral. Universidad Autónoma de Barcelona.

Roca M., Márquez C., Sanmartí N. (2013) Las preguntas de los alumnos: una propuesta de análisis. Enseñanza de las Ciencias, 31(1), 95-114. https://doi.org/10.5565/rev/ec/v31n1.603

Rodríguez Moreno J., Pro Bueno A. y Molina Jaén M. D. (2018). Opinion de los docentes sobre el tratamiento de las competencias en los libros de texto de Ciencias de la Naturaleza en Educacion Primaria. Revista Eureka sobre Enseñanza y Divulgación de las $\begin{array}{llll}\text { Ciencias } & 18 & \text { (3), } & 3102 .\end{array}$ https://doi.org/10.25267/Rev_Eureka_ensen_divulg_cienc.2018.v15.i3.3102

Royo M. A., Rodríguez F. (2015) Publicidad alimentaria y salud. Estado de la situación en España. Mediterráneo Económico, 27, 319-330.

Segovia M. J., Villares J. M. (2010) El desayuno en la infancia: más que una buena costumbre. Acta Pediátrica Española, 68(8), 403-408.

Tornero B., Ramaciotti A., Truffello A., Valenzuela F. (2015) Nivel cognitivo de las preguntas que formulan las educadoras de párvulos. Educación y Educadores, 18(2), 261-283. https://doi.org/10.5294/edu.2015.18.2.5

Torres-García M, Marrero-Montelongo M, Navarro-Rodríguez C, Gavidia V (2018) ¿Cómo abordan los textos de Educación Primaria la competencia en alimentación y actividad física? Revista Eureka sobre Enseñanza y Divulgación de las Ciencias 15(1), 1103. https://doi.org/10.25267/Rev_Eureka_ensen_divulg_cienc.2018.v15.11.1103

Wartofsky M. (1968) Conceptual Foundations of Scientific Thought: An introduction to the philosophy of science. New York: The Macmillan Company.

Wells G. (2002) The Role of Dialogue in Activity Theory. Mind. Culture and Activity, 9, 43-66. https://doi.org/10.1207/S15327884MCA0901_04

Woodward C. (1992) Raising and answering questions in primary science: some considerations. Evaluation and Research in Education, 6(2,3), 145-153. https://doi.org/10.1080/09500799209533324 
Anexo I. Listado completo de libros analizados

\begin{tabular}{|c|c|c|}
\hline Editorial & Curso & Referencia \\
\hline \multirow{6}{*}{$A N A Y A$} & $1^{\circ}$ & ISBN: 978-84-698-3868-6 \\
\hline & $2^{\circ}$ & ISBN: 978-84-698-3875-4 \\
\hline & $3^{\circ}$ & ISBN: 978-84-698-4224-9 \\
\hline & $4^{\circ}$ & ISBN: 978-84-698-0754-5 \\
\hline & $5^{\circ}$ & ISBN: 978-84-698-4383-3 \\
\hline & $6^{\circ}$ & ISBN: 978-84-698-8090-8 \\
\hline \multirow{6}{*}{ EDEBÉ } & $1^{\mathrm{o}}$ & ISBN: 978-84-683-1409-9 \\
\hline & $2^{\circ}$ & ISBN: 978-84-683-2102-8 \\
\hline & $3^{\circ}$ & ISBN: 978-84-683-1461-7 \\
\hline & $4^{\circ}$ & ISBN: 978-84-683-2042-7 \\
\hline & $5^{\circ}$ & ISBN: 978-84-683-1462-4 \\
\hline & $6^{\circ}$ & ISBN: 978-84-683-2043-4 \\
\hline \multirow{6}{*}{ EDELVIVES } & $1^{\circ}$ & ISBN: 978-84-263-9299-2 \\
\hline & $2^{\circ}$ & ISBN: 978-84-263-9591-7 \\
\hline & $3^{\circ}$ & ISBN: 978-84-263-9319-7 \\
\hline & $4^{\circ}$ & ISBN: 978-84-263-9623-5 \\
\hline & $5^{\circ}$ & ISBN: 978-84-263-9363-0 \\
\hline & $6^{\circ}$ & ISBN: 978-84-263-9653-2 \\
\hline \multirow{6}{*}{ SANTILLANA } & $1^{\circ}$ & ISBN: 978-84-680-1163-9 \\
\hline & $2^{\circ}$ & ISBN: 978-84-680-1485-2 \\
\hline & $3^{\circ}$ & ISBN: 978-84-680-4736-2 \\
\hline & $4^{\circ}$ & ISBN: 978-84-680-2919-1 \\
\hline & $5^{\circ}$ & ISBN: 978-84-680-2554-4 \\
\hline & $6^{\circ}$ & ISBN: 978-84-680-2920-7 \\
\hline \multirow{6}{*}{ VICENS VIVES } & $1^{\circ}$ & ISBN: 978-84-682-5474-6 \\
\hline & $2^{\circ}$ & ISBN: 978-84-682-5487-6 \\
\hline & $3^{\circ}$ & ISBN: 978-84-682-2070-3 \\
\hline & $4^{\circ}$ & ISBN: 978-84-682-2840-2 \\
\hline & $5^{\circ}$ & ISBN: 978-84-682-1504-4 \\
\hline & $6^{\circ}$ & ISBN: 978-84-682-1558-7 \\
\hline
\end{tabular}

